Annals of Pure and Applied Mathematics

Vol. 14, No. 2, 2017, 307-312

ISSN: 2279-087X (P), 2279-0888(online)

Published on 23 September 2017

www.researchmathsci.org

DOI: http://dx.doi.org/10.22457/apam.v14n2a13

Annals of

Pure and Applied

Mathematics

\title{
On the Infinitude of Covering Systems with Least Modulus Equal to 2
}

\author{
Nechemia Burshtein
}

117 Arlozorov Street, Tel Aviv 6209814, Israel

Email: anb17@ netvision.net.il

Received 19 September 2017; accepted 22 September 2017

\section{Dedicated to the memory of the late Prof. Paul Erdös friend and mentor}

Abstract. A finite set of residue classes $a_{i}\left(\bmod n_{i}\right)$ with $1<n_{1}<n_{2}<\cdots<n_{s}$ is called a covering system of congruences if every integer satisfies at least one of the congruences $x \equiv a_{i}\left(\bmod n_{i}\right)$. An example is the set $\{0(\bmod 2), 1(\bmod 3), 3$ $(\bmod 4), 5(\bmod 6), 9(\bmod 12)\}$. A covering system all of whose moduli are odd called an odd covering system is a famous unsolved conjecture of Erdös and Selfridge. In this paper, we establish that there exist infinitely many even covering systems in which the least modulus is 2 and all other moduli are even. In each such even covering system, the number of the moduli and their prime factors are determined. Moreover, we construct a covering system with nine moduli, the smallest modulus is 2 , and the lcm of the moduli is divisible by only the primes 2 and 5. With the smallest modulus 2, this is an attempt in the direction of constructing covering systems none of whose moduli is a product of the prime 3 .

Keywords: Congruences, covering systems, exactly covering systems

AMS Mathematics Subject Classification (2010): 11B25, 11A07

\section{Introduction}

The concept of Covering systems was introduced and developed by Erdös. Many authors and numerous articles have been written on this interesting and non-trivial topic, and quite a wide literature exists on the subject.

We begin with some definitions and notations.

Definition 1.1. The system of congruences

$$
x \equiv a_{i}\left(\bmod n_{i}\right), \quad 1<n_{1}<n_{2}<\cdots<n_{t}, \quad 0 \leq a_{i}<n_{i}
$$

is called a Covering System (abbreviated CS), if every integer satisfies at least one of the congruences (1). 
Nechemia Burshtein

Definition 1.2. The system of congruences

$$
x \equiv a_{i}\left(\bmod n_{i}\right), \quad 1<n_{1} \leq n_{2} \leq \cdots \leq n_{k}, \quad 0 \leq a_{i}<n_{i}
$$

is called an Exactly Covering System (abbreviated ECS), if every integer satisfies exactly one of the congruences (2).

A system of congruences corresponding to any of the above two definitions, will be denoted as a set of ordered pairs of integers of the form $(a, n)$, where $a$ is the residue class and $n$ is the modulus.

The least common multiple of the moduli in a CS/ECS will be denoted by LCM.

The smallest and simplest example of a CS is the following set of ordered pairs

$$
\{(0,2),(0,3),(1,4),(5,6),(7,12)\} .
$$

Hereafter, we shall refer to the set (3) as the basic CS.

A necessary condition [7] on the moduli $n_{1}, n_{2}, \ldots, n_{t}$ of a CS is

$$
\sum_{i=1}^{t} \frac{1}{n_{i}}>1 \text {. }
$$

Definition 1.3. An ECS, in which for every value $m$ there are at most $M$ moduli which are equal to $m$, will be called an $\operatorname{ECS}(M)$.

It is well known $[8,7]$, that the moduli $n_{1} \leq n_{2} \leq \cdots \leq n_{k}$ of any ECS satisfy:

$$
\sum_{i=1}^{k} \frac{1}{n_{i}}=1 \quad\left(n_{i}, n_{j}\right)>1
$$

and

$$
n_{k-1}=n_{k} \text {. }
$$

Condition (5) implies moreover that $M=1$ is impossible.

The simplest examples of ECS's are the following sets $\{(0,2),(1,2)\}, \quad\{(0,2),(1,4),(3,4)\}, \quad\{(0,2),(1,4),(3,8),(7,8)\}$.

Hereafter, we shall refer to sets described in (6) as basic ECS's. The moduli of each set in (6) satisfy the conditions in (4) and (5).

The basic ECS's in (6) are also known in the literature as Natural ECS's (abbreviated NECS's). As for $\operatorname{ECS} s(M)$ we also have accordingly $\operatorname{NECS's~}(M)$. Many results on ECS's and NECS's may also be found in $[1,2,3,4,10,16,17$, $18,20,21]$.

We shall now cite some known results, in particular those concerned with the least modulus $n_{1}$ of a CS. Erdös asked whether there are CS's in which $n_{1} \geq R$ for arbitrary $R$. Over the last 60 years the value $R$ increased. In 1968, Churchhouse [6] using a computer found CS's with $n_{1}=2,3, \ldots, 9$. In 1971, Krukenberg [13] gave examples of CS's for all values $n_{1}$ up to 18 inclusive. 
On the Infinitude of Covering Systems with Least Modulus Equal to 2

Choi [5] found a CS with $n_{1}=20$. The current record belongs to Owens [15] with $n_{1}=42$. Hough showed the amazing result [12] that $n_{1}$ is at most $10^{16}$. For this achievement he won the 2017 David P. Robbins prize.

Other results may also be found for instance in $[9,13,16,19,21]$.

\section{On the infinitude of even CS's when the least modulus is 2}

In this section, we exhibit a connection between an ECS and a CS in each of which the smallest modulus is 2. First, this is illustrated in two self-contained examples, namely Example 2.1 and Example 2.2 using two of the basic ECS's in (6) and the basic CS in (3). Secondly, the pattern described in these examples enables us to establish the general case, i.e., the existence of infinitely many even CS's with smallest modulus 2. This is done in Theorem 2.1.

Example 2.1. The basic ECS in (6) whose moduli are $\{2,4,4\}$ combined with the basic CS in (3) whose moduli are $\{2,3,4,6,12\}$ yield the set of seven moduli

$$
\{2,4, \quad 4 \cdot 2,4 \cdot 3,4 \cdot 4,4 \cdot 6,4 \cdot 12\},
$$

which are even, distinct and $\sum_{i=1}^{7} \frac{1}{n_{i}}>1$. The respective $\mathrm{CS}$ is

$$
\{(0,2),(3,4),(5,8),(5,12),(9,16),(9,24),(1,48)\} \text {. }
$$

Example 2.2. The basic ECS in (6) whose moduli are $\{2,4,8,8\}$ combined with the basic CS in (3) whose moduli are $\{2,3,4,6,12\}$ yield the set of eight moduli

$$
\{2,4,8, \quad 8 \cdot 2,8 \cdot 3,8 \cdot 4,8 \cdot 6,8 \cdot 12\},
$$

which are even, distinct and $\sum_{i=1}^{8} \frac{1}{n_{i}}>1$. The respective CS is

$$
\{(0,2),(3,4),(1,8),(5,16),(13,24),(29,32),(45,48),(77,96)\} \text {. }
$$

Example 2.1 and Example 2.2, clearly show the pattern which connects a basic ECS with the basic CS. The general case will now be established for all basic ECS's of the form described in (6) combined with the basic CS in (3).

Theorem 2.1. For each and every value $t \geq 1$, the set of ordered pairs

$$
\left\{(0,2),(1,4),(3,8),(7,16), \ldots,\left(2^{t-1}-1,2^{t}\right),\left(2^{t}-1,2^{t}\right)\right\}
$$

represents a basic ECS. Let $t \geq 1$ be any fixed value. Then:

(i) The set

$$
\left\{2,4,8,16, \ldots, 2^{t}, \quad 2^{t} \cdot 2,2^{t} \cdot 3,2^{t} \cdot 4,2^{t} \cdot 6,2^{t} \cdot 12\right\}
$$

is a set of moduli of a CS.

(ii) There are infinitely many CS's whose moduli satisfy (8).

(iii) All the moduli in (8) are even and distinct.

(iv) In (8), the LCM is $2^{t} \cdot 12$ whose prime divisors are 2 and 3. 
Nechemia Burshtein

(v) The number $k$ of the moduli in (8) is equal to $k=t+5$.

Proof: For (i), recall from (3) the moduli $\{2,3,4,6,12\}$ of the basic $\mathrm{CS}$ whose $\mathrm{LCM}=12$. It is noted that if the integers $1-12$ are covered, then all the integers are covered, and the set of moduli is a CS. As for the moduli 2, 4 in (3), the moduli $2 \cdot 2^{t}, 4 \cdot 2^{t}$ in (8) and their values $a_{i}$ yield up to the new $\mathrm{LCM}=12 \cdot 2^{t}$ respectively six and three integers all of which are distinct with no overlaps. The other three moduli in (8), namely $3 \cdot 2^{t}, 6 \cdot 2^{t}$ and $12 \cdot 2^{t}$ and their appropriate values $a_{i}$, each yields exactly one integer up to $12 \cdot 2^{t}$, whereas all other obtained integers are overlaps. Thus, all twelve integers are covered up to $12 \cdot 2^{t}$ as required, and (8) is a set of moduli of a CS as asserted.

As for (ii), each value $t \geq 1$ in (7) yields a basic ECS. Hence, there are infinitely many basic ECS's. Then, for each and every value $t \geq 1$ there exists a CS. Thus, there exist infinitely many CS's whose moduli satisfy (8).

The statements in (iii), (iv) and (v) follow directly from (8).

This completes the proof of Theorem 2.1.

The following Corollary 2.1 follows from Theorem 2.1.

Corollary 2.1. The sum of the reciprocals of the five moduli in (3) is $\sum_{i=1}^{5} \frac{1}{n_{i}}=\frac{4}{3}$. The sum of the reciprocals of the $(t+5)$ moduli in (8) is equal to

$$
\sum_{i=1}^{t+5} \frac{1}{n_{i}}=\frac{2^{t}-1}{2^{t}}+\frac{1}{2^{t}} \cdot \frac{4}{3}=\frac{3 \cdot 2^{t}+1}{3 \cdot 2^{t}}=1+\frac{1}{3 \cdot 2^{t}} .
$$

For arbitrarily large $t$, it follows that the reciprocals of the moduli of the CS in (8) have a sum which is as close to 1 as we wish, but is never equal to 1 .

Remark 2.1. In Theorem 2.1, we have used the basic CS having the five moduli 2, 3, 4, 6, 12. As the least modulus increases, the larger are the number of the moduli and so are their prime factors. Evidently, for the purposes of Theorem 2.1, any known CS will suffice instead of the basic CS. However, constructing a CS union of a basic ECS and the basic CS, has its advantages primarily in (i) the smallest number of obtained moduli, and (ii) their largest prime divisor which is $p=3$.

\section{On a CS whose least modulus is 2 and the LCM is $2^{4} \cdot 5$}

In the literature, all CS's with least modulus 2, have LCM's which are divisible by $p=3$. Hough and Nielsen have shown that every CS has a modulus divisible by either 2 or 3 .

In this section, we exhibit Example 3.1 i.e., a CS of nine moduli containing the least modulus 2 , the modulus 5 , and the LCM $2^{4} \cdot 5=80$. This is the first CS none of whose moduli is divisible by $p=3$. 
On the Infinitude of Covering Systems with Least Modulus Equal to 2

Example 3.1. The following set of nine ordered pairs is a CS in which except for 1 , the nine moduli are all the divisors of 80 .

$\{(1,2),(0,4),(0,5),(2,8),(6,10),(6,16),(14,20),(22,40),(78,80)\}$.

A CS with least modulus 2 and the only odd modulus 5 does not exist when $k<9$. Hence, Example 3.1 is a CS whose number of mosuli $k=9$ is minimal. For any other CS of the same nature, it follows that $k>9$. Moreover, as mentioned earlier, Example 3.1 now implies that there exists at least one CS which does not contain a modulus divisible by 3 , but rather, moduli divisible by 2 .

Final Remark. In view of Example 3.1, we may now presume that for primes $p \geq 7$, there exists a CS containing the least modulus 2 and the $\mathrm{LCM}=2^{r} \cdot p$.

\section{REFERENCES}

1. N.Burshtein, Exactly covering systems of congruences, Ph.D. thesis, University of Tel Aviv, 1974.

2. N.Burshtein, On natural exactly covering systems of congruences having moduli occurring at most $M$ times, Discrete Mathematics, 14 (3) (1976) $205-214$.

3. N.Burshtein, On natural exactly covering systems of congruences having moduli occurring at most twice, Journal of Number Theory, 8 (3) (1976) $251-259$.

4. N.Burshtein and J.Schönheim, On exactly covering systems of congruences having moduli occurring at most twice, Czechoslovak Mathematical Journal, 24 (3) (1974) 369-372.

5. S.L.G.Choi, Covering the set of integers by congruence classes of distinct moduli, Math. Comp., 25 (1971) 885 - 895.

6. R.F.Churchhouse, Covering sets and systems of congruences, Computers in Mathematical Research, North-Holland, Amsterdam, 1968, pp. 20 - 36.

7. P.Erdös, On a problem concerning congruence systems, Mat. Lapok 3 (1952) $122-128$.

8. P.Erdös, Számelméleti megjegyzések iv, Mat. Lapok 17 (1962) 228 - 255.

9. P.Erdös and R.L.Graham, Old and new problems and results in Combinatorial Number Theory, Monographie $\mathrm{n}^{0} 28$ de L'Enseignement Mathématique Université de Genève, Imprimerie Kundig Genève, 1980.

10. A.S.Fraenkel, A characterization of exactly covering congruences, Discrete Mathematics 4 (1973) 359 - 366.

11. D.J.Gibson, A covering system with least modulus 25, Math. Comp., 78 (266) (2009) 1127 - 1146.

12. B.Hough, Solution of the minimum modulus problem for covering systems, Annals of Mathematics, 181 (1) (2015) 361 - 382. 
Nechemia Burshtein

13. C.E.Krukenberg, Covering sets of the integers, Ph.D. thesis, University of Illinois at Urbana-Champaign, 1971.

14. P.Nielsen, A covering system whose smallest modulus is 40, Journal of Number Theory, 129 (3) (2009) 640 - 666.

15. T.Owens, A covering system with minimum modulus 42, Master's thesis, Brigham Young University at Provo, 2014.

16. Š.Porubský and J.Schönheim, Covering systems of Paul Erdös: past, present and future, in: Paul Erdös and his Mathematics I (edited by G. Halász, L.Lovász, M. Simonvits, V. T. Sós), Bolyai Soc. Math Studies 11, Budapest, 2002, pp. $581-627$.

17. J.Schönheim and N.Burshtein, On a conjecture concerning exactly covering systems of congruences, Israel Journal of Mathematics, 8 (1) (1970) 28 - 29.

18. R.J.Simpson, Exact coverings of the integers by arithmetic progressions, Discrete Math., 59 (1986) 181 - 190.

19. R.J.Simpson and D.Zeilberger, Necessary conditions for distinct covering systems with square-free moduli, Acta. Arith., 59 (1) (1991) 59 - 70.

20. S.Znám, On exactly covering systems of arithmetic sequences, Math Ann., 180 (1969) $227-232$.

21. S.Znám, A survey of covering systems of congruences, Acta Math., Universitatis Comenianae 40 - 41 (1982) 59 - 78. 